\title{
Triple tetracycline (Deteclo) in the treatment of chlamydial infection of the female genital tract
}

\author{
M. A. WAUGH AND K. C. NAYYAR \\ From the Department of Sexually Transmitted Diseases, General Infirmary at Leeds
}

SUMmaRY The efficacy of treating genital Chlamydia in women using Deteclo $300 \mathrm{mg}$ twice daily for seven and 21 days has been studied. Forty-four patients were treated for seven days and 20 for 21 days. Seven days of treatment was as effective in eliminating Chlamydia trachomatis from the female genital tract as 21 days. The reproducibility of the technique of isolation of $C$. trachomatis was assessed by delaying treatment in 10 patients for up to 156 days and finding $C$. trachomatis still present.

\section{Introduction}

Since the isolation of Chlamydia trachomatis in yolk-sac culture (Jones et al., 1959) evidence suggesting its pathogenicity in non-specific urethritis (NSU) has gained strength. It has been isolated in 12 to $31 \%$ of women attending venereal disease clinics (Hilton et al., 1974; Hobson et al., 1974; Oriel et al., 1974; Burns et al., 1975; Nayyar et al., 1976). It has been shown that three-quarters of the women who are chlamydia-positive are asymptomatic (Nayyar et al., 1976), but these women are presumed to have non-specific genital infection (NSGI). In view of the potential infectivity and recurrence of NSU in the male partner, as well as the possible complications of pelvic inflammatory disease in these patients, the authors believe that women who harbour Chlamydia should be treated, although there is little information on the effect of antibiotic treatment on chlamydial infection of the female genital tract.

This study was made to investigate the efficacy of tetracyclines and their optimal dosage in treatment of NSGI in chlamydia-positive women.

\section{Patients and methods}

The patients involved in this investigation attended the Sexually Transmitted Diseases Clinic at Leeds General Infirmary between October 1975 and July 1976. In all cases $C$. trachomatis was cultured on untreated McCoy cells.

Address for reprints: M. A. Waugh, Department of Sexually Transmitted Diseases, General Infirmary at Leeds, Leeds LS1 3EX

Received for publication 3 August 1976
Infection with gonorrhoea, trichomoniasis, and candidiasis was excluded by the following methods. Urethral and cervical smears were stained with Gram stain and examined for the presence of Gramnegative diplococci. Specimens for Neisseria gonorrhoeae from the urethra and cervix were inoculated on to modified Thayer-Martin medium and incubated at $37^{\circ} \mathrm{C}$ in an atmosphere of air plus $5 \%$ $\mathrm{CO}_{2}$. High vaginal smears were stained with Gram stain and examined for the presence of Grampositive organisms and mycelia. High vaginal specimens were also inoculated on to Sabouraud's medium. A wet preparation of the vaginal specimen was examined for the presence of Trichomonas vaginalis and the specimen was also cultured in Feinberg-Whittington medium.

Specimens for $C$. trachomatis were collected by rotating a cotton wool swab in the cervical canal and then breaking off the tip into a small screwcapped bottle containing transport medium (McCoy cell growth medium with $0.5 \%$ glucose and $10 \%$ sorbitol). The specimens were stored at a temperature of $4^{\circ} \mathrm{C}$ for three hours before being transferred to the virology laboratory. The method used for the isolation of $C$. trachomatis has been described previously (Nayyar et al., 1976).

\section{Treatment}

Two groups of patients were studied. The first comprised patients attending in the first two months of the study, and the second comprised patients attending in the last four months. Both groups were given chlortetracycline hydrochloride $115.4 \mathrm{mg}$, tetracycline hydrochloride $115.4 \mathrm{mg}$, demeclo- 
cycline hydrochloride $69.2 \mathrm{mg}$ (Deteclo). Those in Group 1 took one tablet twice daily for 21 days, those in Group 2 took one tablet twice daily for seven days.

\section{Reproducibility}

To assess the reproducibility of the technique of isolation of $C$. trachomatis, treatment was delayed in some (arbitrarily chosen) patients, and tests for C. trachomatis were repeated before starting treatment. Ten entered this group; all were found to be chlamydia-positive on repeated investigation, six at seven days, and one each at $14,21,42$, and 156 days.

\section{Follow-up and results}

In this study 75 chlamydia-positive patients were investigated. Their ages ranged from 17 to 28 years (mean 21.9) in Group 1, and from 17 to 44 years (mean 22.1) in Group 2. Eleven patients defaulted and were excluded from the study.

In Group 1,20 patients were treated. In 10 patients chlamydial cultures were taken seven days after starting treatment, and in all patients cultures were taken seven days after treatment had finished. All gave negative results. Two patients were seen 42 and 120 days after the end of treatment and still had negative chlamydial culture results.

In Group 2, 44 patients were treated. Cultures were taken for Chlamydia at the end of treatment and 21 days later. All gave negative results. Four of 44 patients were examined again at 56 days and were still chlamydia-negative.

\section{Discussion}

This study shows that $C$. trachomatis persisted in the absence of any treatment in six patients for seven days, and in one each for 14, 21, 42, and 156 days. It shows that the reproducibility of our technique was quite satisfactory, and indicates that if no antibiotic treatment is given, $C$. trachomatis persists in the female genital tract.

Triple tetracycline was successful in eradicating chlamydia whether given for seven or 21 days. These findings are consistent with those of Jawetz (1969) who reported that tetracyclines suppress chlamydial growth. The only similar study of treatment with tetracyclines of chlamydia-positive women is that of Oriel et al. (1975) who treated 24 patients with a semi-synthetic tetracycline, minocycline $100 \mathrm{mg}$ twice daily for 21 days, all becoming chlamydianegative by the end of treatment. In view of our findings we plan to expand this study by a longer length of surveillance after treatment.

We wish to thank Dr M. H. Hambling, Department of Virology, Public Health Laboratory Service, Leeds for his technical assistance.

\section{References}

Burns, D. C. MacD., Darougar, S., Thin, R. N., Lothian, L., and Nicol, C. S. (1975). Isolation of Chlamydia from women attending a clinic for sexually transmitted disease. British Journal of Venereal Diseases, 51, 314-318.

Hilton, A. L., Richmond, S. J., Milne, J. D., Hindley, F., and Clarke S. K. R. (1974). Chlamydia $A$ in the female genital tract. British Journal of Venereal Diseases, 50, 1-10.

Hobson, D., Johnson, F. W. A., Rees, E., and Tait, I. A. (1974). Simplified method for diagnosis of genital and ocular infections with Chlamydia. Lancet, 2, 555-556.

Jawetz, E. (1969). In Advances in Pharmacology and Chemotherapy, p. 253. Edited by S. Garattini, A. Goldin, F. Hawking, and I. J. Kopin. Academic Press: New York and London.

Jones, B. R., Collier, L. H., and Smith, C. H. (1959). Isolation of virus from inclusion blennorrhoea. Lancet, 1, 902-905.

Nayyar, K. C., O'Neill, J. J., Hambling, M. H., and Waugh, M. A. (1976). Isolation of Chlamydia trachomatis from women attending a clinic for sexually transmitted diseases. British Journal of Venereal Diseases, 52, 396-398.

Oriel, J. D., Powis, P. A., Reeve, P., Miller, A., and Nicol, C. S. (1974) Chlamydial infections of the cervix. British Journal of Vene real Diseases, 50, 11-16.

Oriel, J. D., Reeve, P., and Nicol, C. S. (1975). Minocycline in the treatment of nongonococcal urethritis: its effect on Chlamydia trachomatis. Journal of the American Venereal Disease Association, 2, 17-22. 